\title{
Silica Glass Toughened by Consolidation of Glassy Nanoparticles
}

\author{
Yanming Zhang, Liping Huang, Yunfeng Shi*
}

Department of Materials Science and Engineering, Rensselaer Polytechnic Institute, $1108^{\text {th }}$ Street, Troy, New York 12180, USA

*shiy2@rpi.edu 


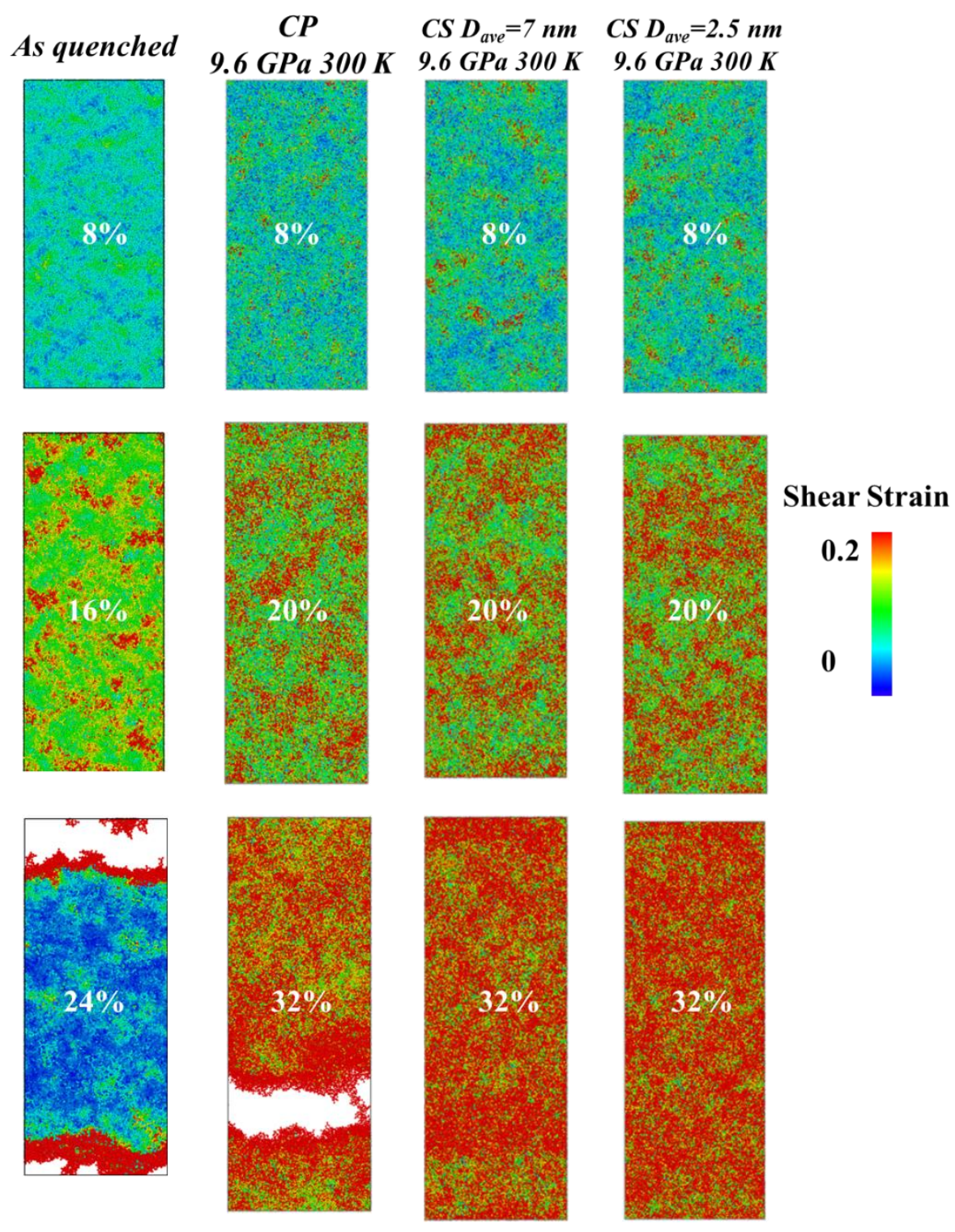

Figure S1. Shear strain distribution for as quenched glass, compressed silica (CP) and consolidated silica (CS) during uniaxial tension test. The applied pressure to prepare compressed silica and consolidated silica is $9.6 \mathrm{GPa}$ and temperature is $300 \mathrm{~K}$. Homogeneous deformation can be observed in consolidated silica and elastic deformation proceeding brittle fracture can be observed for as quenched silica. 


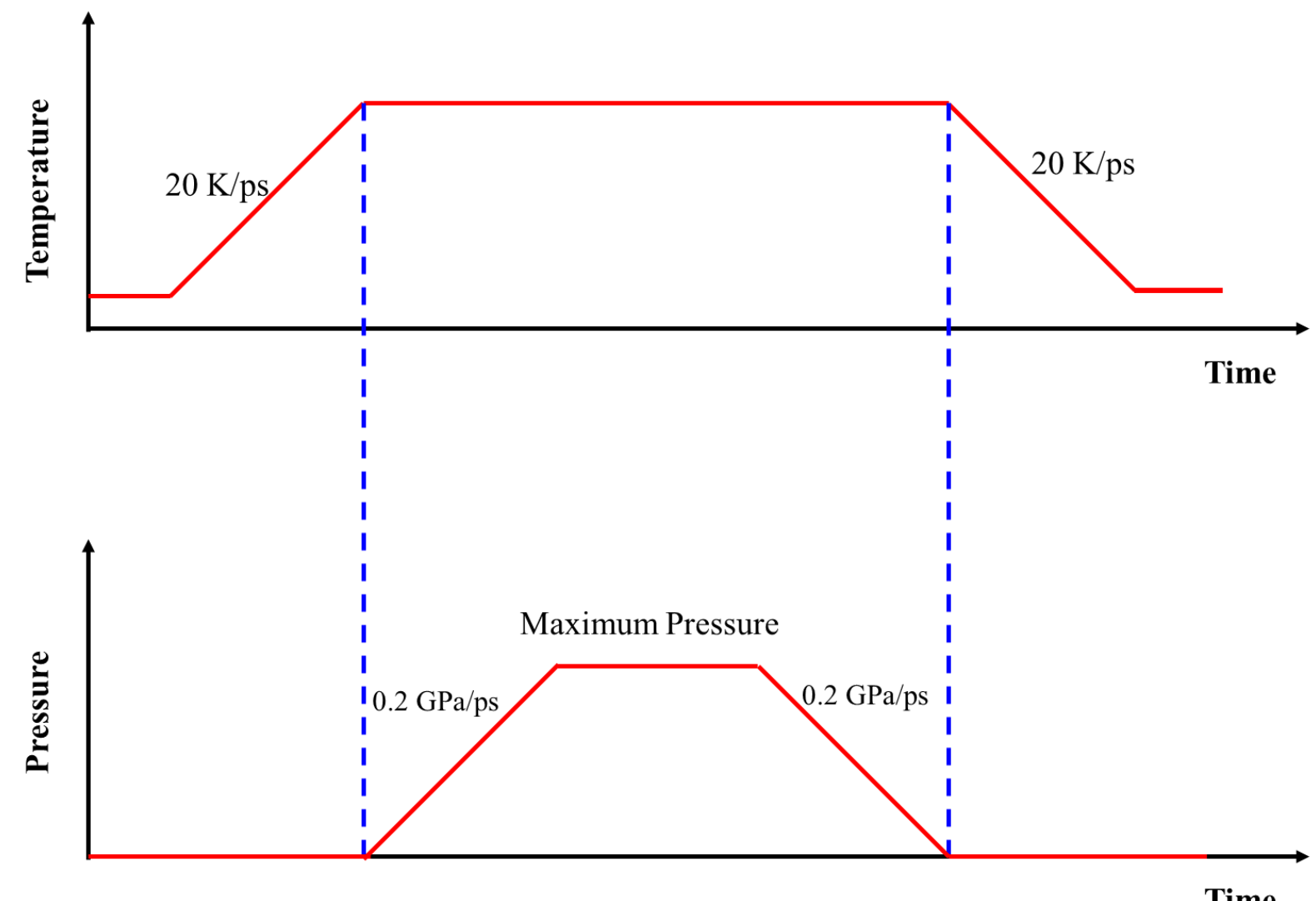

Figure S2. Schematic diagram of the consolidation route. Consolidation process can be carried out at different temperatures ( $300 \mathrm{~K}$ to $2100 \mathrm{~K}$ ) and pressures (1.2 GPa to $19.2 \mathrm{GPa}$ ). Note the heating and cooling rate is fixed at $\sim 20 \mathrm{~K} / \mathrm{s}$. Applied pressure is hold for $1.6 \mathrm{~ns}$ and then released at elevated temperature. Pressure ramping and releasing rate is fixed at $0.2 \mathrm{GPa} / \mathrm{ps}$. 


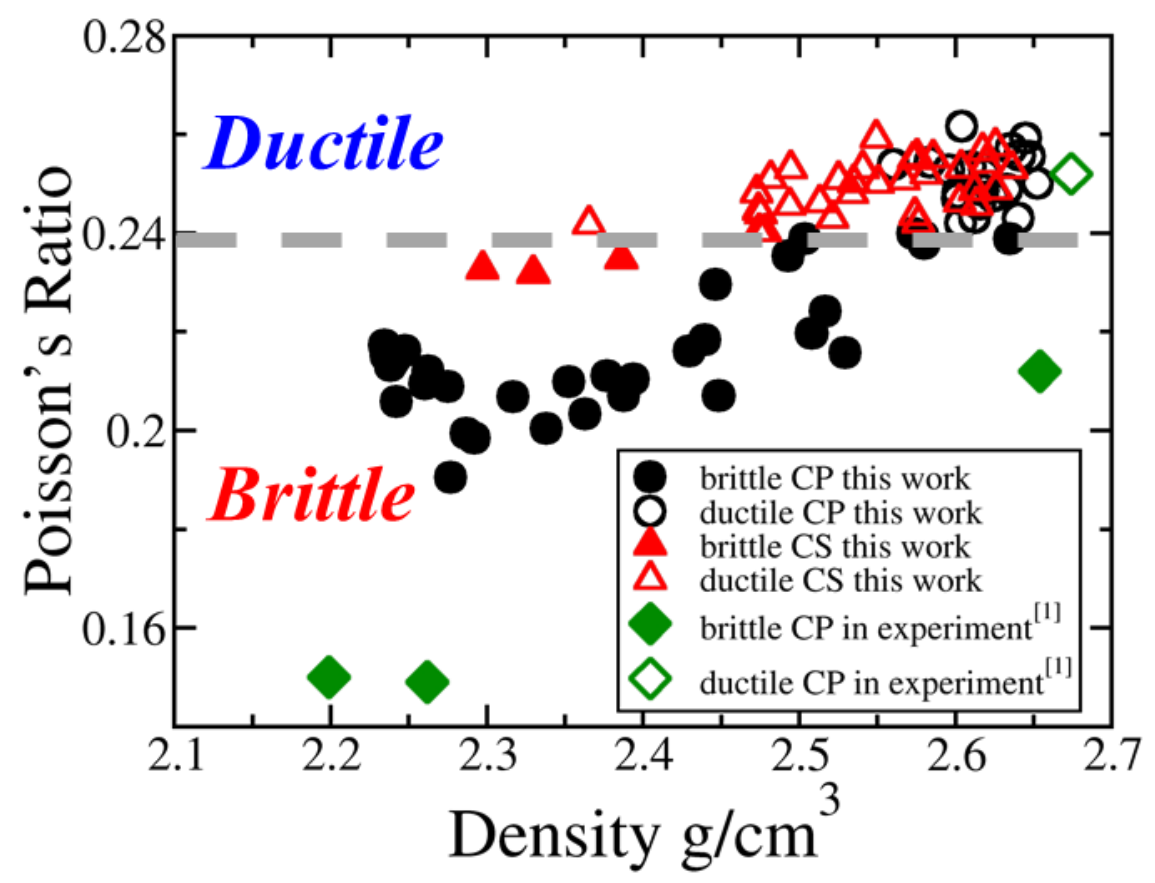

Figure S3. The mapping of CP samples (filled or unfilled circles), CS samples (filled or unfilled triangles), as well as experimental densified silica (ref 34) (filled or unfilled diamonds) in the domain of the Poisson's ratio and density at ambient condition. All ductile samples are represented by unfilled symbols. Simulation results are in general consistent with experimental observations, with a critical Poisson's ratio for BTD transition at around 0.24. 


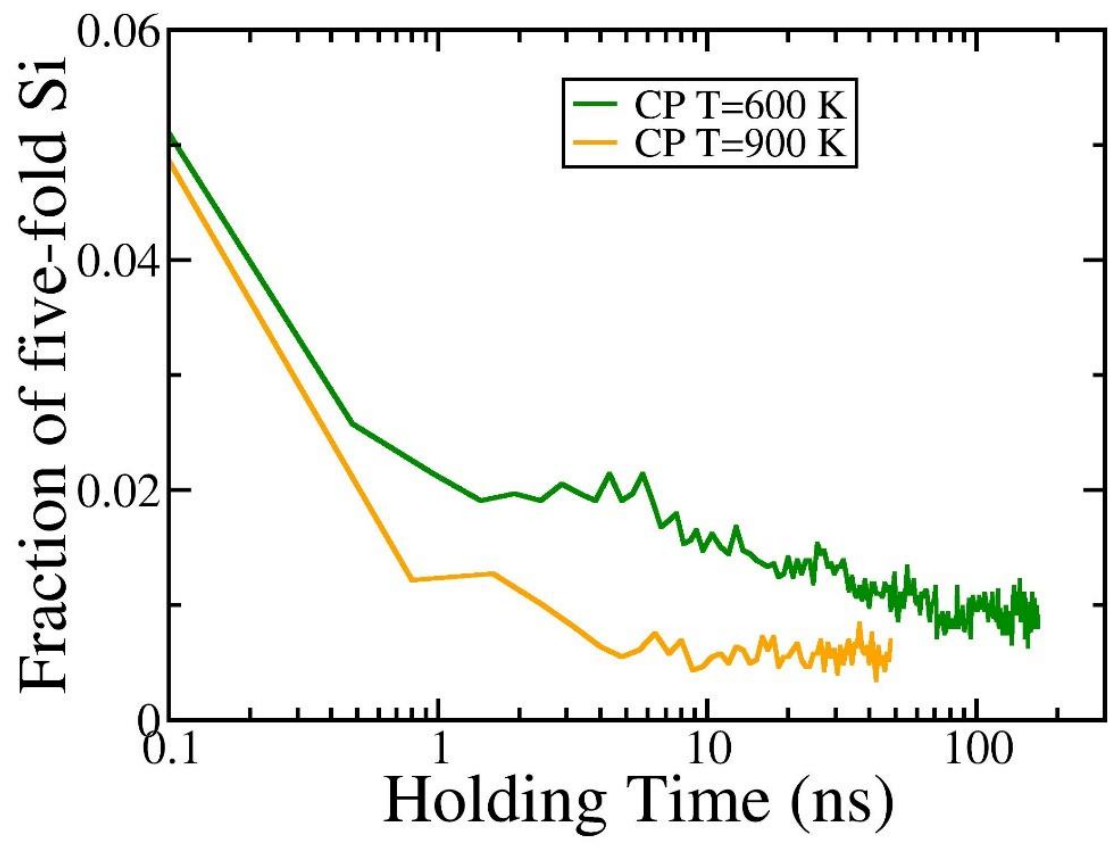

Figure S4. Evolution of five-fold silicon atoms population (holding time in logarithmic scale) when annealed at $600 \mathrm{~K}$ or $900 \mathrm{~K}$ under zero pressure for compressed silica glass $(\mathrm{CP})$. The size of the CP sample is $\sim 10000$ atoms to speed up the simulation for such long time annealing. The corresponding stable population of five-fold silicon retains at $\sim 0.5 \%, \sim 1 \%$ when annealing at 900 $\mathrm{K}$ and $600 \mathrm{~K}$. It should be emphasized that for as quenched silica glass without any pressure treatment, the population of five-fold silicon is almost zero when annealing at $600 \mathrm{~K}$ and $900 \mathrm{~K}$. 

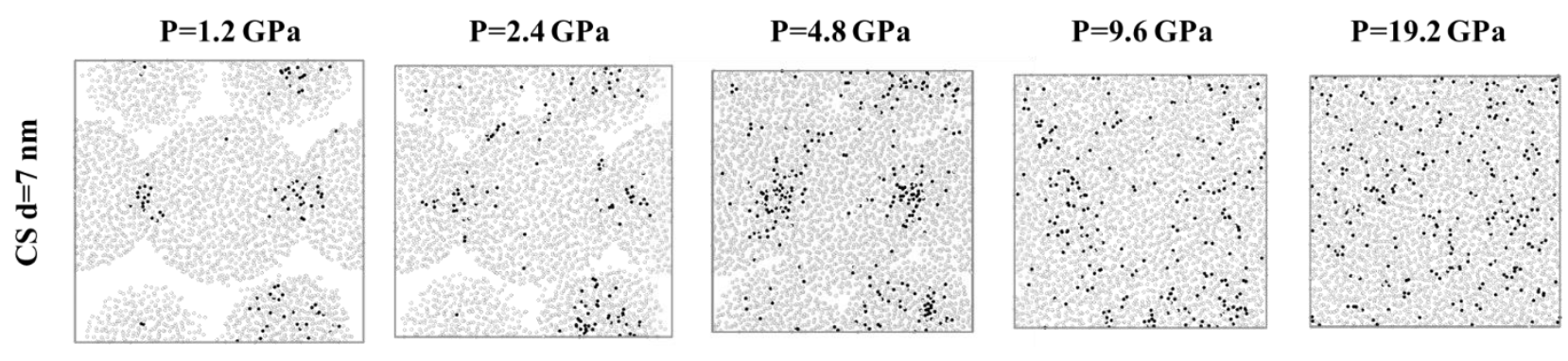

Figure S5. Distribution of five-fold silicon atoms within $1 \mathrm{~nm}$ slice for silica glass prepared with $\sim 7 \mathrm{~nm}$ sized nanoparticles under different consolidation pressure. The black particles represent five-fold silicon and gray particles represent four-fold silicon atoms. Oxygen atoms are omitted for clarity. It can be seen five-fold silicon atoms are near the contact regions under low consolidation pressure, thus heterogeneously distributed. Under high consolidation pressure, the distribution becomes more homogeneous. 

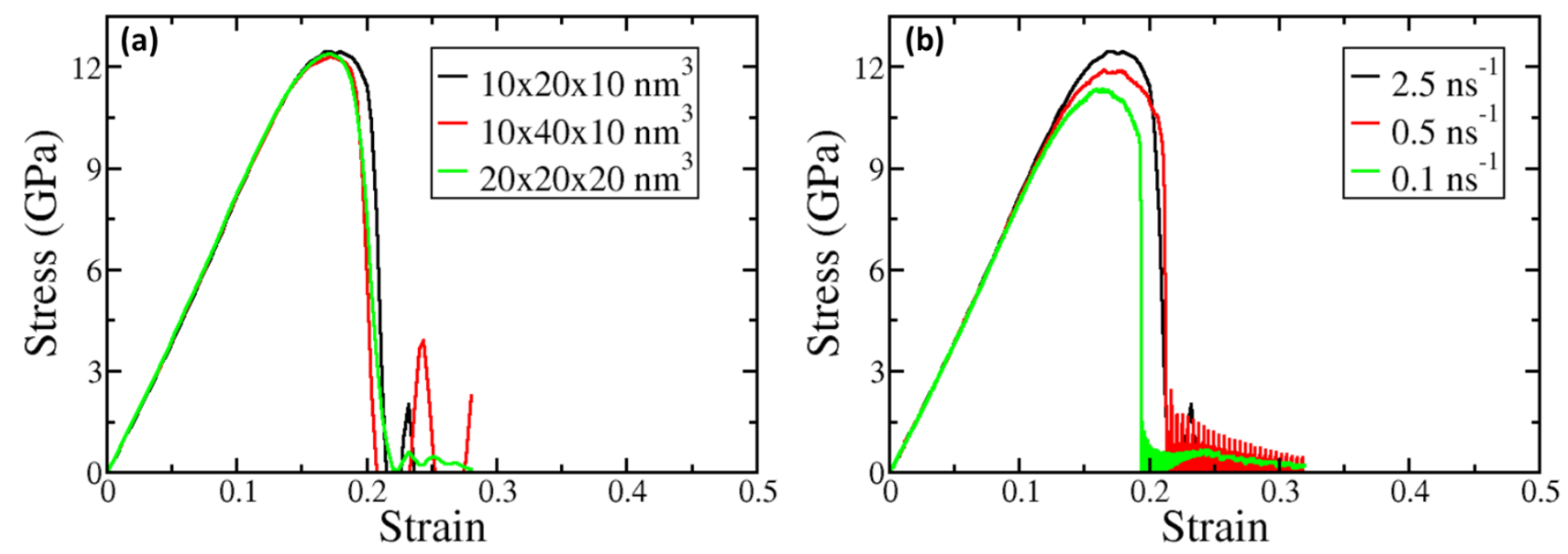

Figure S6. Stress-strain curves under uniaxial tension test at different (a) sample sizes (b) strain rates. To save computational cost and avoid artificial ductility, the sample size we selected is $10 \times 20 \times 10 \mathrm{~nm}^{3}$ and the strain rate is $2.5 \mathrm{~ns}^{-1}$. 